\title{
Sugestões de Rotas Personalizadas para Carrinheiros na Coleta Seletiva de Materiais Recicláveis
}

\author{
Maria Vitória R. Oliveira ${ }^{1}$, Islene C. Garcia ${ }^{1}$ \\ ${ }^{1}$ Instituto de Computação - Universidade Estadual de Campinas (UNICAMP) \\ Campinas - SP - Brasil \\ maria.oliveira@students.ic.unicamp.br, islene@unicamp.br
}

\begin{abstract}
Carrinheiros are collectors of recyclable materials that use humanpowered vehicles. Carrinheiro's collection routes can be tiring depending on the paths chosen. Therefore, this work proposes an algorithm for suggesting customizable routes based on three edge costing policies: Less Work Policy, Less Impedance Policy, and Short Distance Policy. This work used the tools osmnx and networkx to construct graphs, geographic data from Open Street Map, and elevations from Topodata. The simulations performed in Simulation of Urban MObility (SUMO) demonstrated that the proposed algorithm could minimize the power applied to push the vehicle, the distance, and the travel time, according to the policy used.
\end{abstract}

Resumo. Os carrinheiros são catadores de materiais recicláveis que utilizam veículos de tração humana. As rotas de coleta dos carrinheiros podem ser cansativas dependendo do trajeto. Sendo assim, este trabalho propõe um algoritmo de sugestão de rotas personalizáveis a partir de três políticas de cálculo de custos de arestas: Política de Menor Trabalho, Política de Menor Impedância e Política de Menor Distância. Utilizou-se as ferramentas osmnx e networkx na construção dos grafos, dados geográficos do Open Street Map e elevações do Topodata. As simulações realizadas no Simulation of Urban MObility (SUMO) demonstraram que o algoritmo proposto pode minimizar a potência aplicada no veículo, a distância e o tempo de percurso, de acordo com a política utilizada.

\section{Introdução}

A Política Nacional de Resíduos Sólidos (PNRS) regulamentou a gestão de Resíduos Sólidos Urbanos (RSU) no Brasil em 2010, estabelecendo diretrizes para a destinação adequada de materiais. De acordo com a PNRS, a gestão integrada de RSU é um conjunto de ações com o objetivo de encontrar soluções para a destinação dos resíduos, considerando as dimensões política, econômica, ambiental, cultural e social. Além disso, a política determina a inclusão dos catadores de materiais reutilizáveis e recicláveis como agentes no processo de desenvolvimento sustentável. Estes profissionais realizam a coleta seletiva, definida como o processo de recolher os resíduos previamente segredados conforme sua constituição ou composição [BRASIL 2010].

Os catadores que utilizam veículos de propulsão humana durante o recolhimento dos materiais geralmente são chamados de carrinheiros. Nesse caso, o roteiro é realizado a pé, empurrando o veículo. Sendo assim, a rota de coleta pode ser extremamente cansativa e/ou demorada para o carrinheiro. Portanto, abordar o problema de roteamento de 
veículos de propulsão humana é importante para proporcionar rotas de coleta eficientes que minimizam o esforço físico ao longo do percurso.

O problema de roteamento de veículos de propulsão humana se divide em dois subproblemas: o primeiro está relacionado à ordenação dos pontos de coleta que devem ser visitados; o segundo está associado à criação de caminhos otimizados entre estes pontos, no mapa geográfico. Dentre os trabalhos que consideram a utilização de veículos de propulsão humana, destacam-se [Vu et al. 2018, Das and Bhattacharyya 2015]. O trabalho [Vu et al. 2018] apresenta um modelo de distribuição geográfica dos pontos de coleta e criação de rotas para diminuir custos financeiros. Além disso, o trabalho [Das and Bhattacharyya 2015] apresenta um esquema de coleta de RSU que minimiza a distância das rotas. Porém, os trabalhos relacionados não levam em consideração o tempo de trajeto e o esforço físico dos carrinheiros.

Este trabalho propõe um algoritmo de sugestão de rotas individuais para carrinheiros que determina a ordem de visita dos pontos de coleta e define os melhores caminhos entre estes pontos. Além disso, propõe-se personalizar os roteiros de coleta de acordo com três políticas: Política de Menor Trabalho (PMT), Política de Menor Impedância (PMI) e Política de Menor Distância (PMD). A política PMT minimiza o trabalho aplicado para empurrar o veículo ao longo do trajeto, a política PMI evita vias que possuem aclives acentuados e a política PMD diminui a distância do roteiro.

As rotas de coleta podem ser personalizadas conforme o objetivo dos carrinheiros e o cenário geográfico, configurando uma das políticas propostas. Por exemplo, se o local de coleta possui declividades acentuadas e/ou o objetivo do catador é diminuir o esforço físico empregado no trajeto, propõe-se utilizar a política PMT ou a PMI. Porém, se o cenário possui baixa variação de altitude e o objetivo do carrinheiro é diminuir a distância percorrida, propõe-se utilizar a política PMD.

O diferencial do algoritmo proposto está na combinação da heurística do Vizinho mais Próximo para determinar a ordem dos pontos de coleta e do algoritmo Shortest Path Faster Algorithm (SPFA), para definir os caminhos no mapa geográfico, considerando a utilização de veículos de tração humana. Outro diferencial é a estratégia empregada pela política PMT, de atualizar o peso do veículo em cada ponto de parada para identificar os melhores caminhos de acordo com o peso do carrinho e as declividades do cenário.

Os cenários de avaliação do algoritmo proposto foram implementados com as ferramentas livres de manipulação de grafos networkx e osmnx. Ademais, os dados geográficos foram obtidos do Open Street Map e a altitude, do Banco de Dados Geomorfométricos do Brasil [Valeriano and Rossetti 2012]. Sendo assim, as políticas PMT, PMI e PMD foram analisadas de acordo com a potência aplicada no veículo, distância e tempo de trajeto. Como resultado, o algoritmo proposto gerou rotas que minimizam a distância e o tempo utilizando a política PMD. Porém, a potência média aplicada no veículo é menor quando as políticas PMT e PMI são empregadas. Além de minimizar a potência, utilizando a política PMT, o algoritmo proporciona rotas que reduzem a distância.

O restante deste documento está organizado da seguinte forma: a Seção 2 apresenta os trabalhos relacionados. Em seguida, a Seção 3 exibe as estratégias e tecnologias na coleta de resíduos. A Seção 4 exibe o algoritmo e as políticas implementadas. Depois, a Seção 5 apresenta os resultados. Por fim, a Seção6 descreve as conclusões do trabalho. 


\section{Trabalhos relacionados}

Diversos trabalhos no contexto da coleta seletiva de materiais recicláveis se concentram no problema de ordenação e distribuição geográfica dos pontos de coleta [Vu et al. 2018, Rathore et al. 2020, Gallardo et al. 2015, Boskovic and Jovicic 2015]. Além de distribuir os pontos de parada no cenário, o trabalho [Vu et al. 2018] propõe um modelo de roteamento de veículos de tração humana e automotores, para minimizar custos financeiros na coleta de resíduos. O modelo proposto apresentou bom desempenho na distribuição geográfica dos pontos de coleta. Porém, as rotas geradas não foram avaliadas. Ademais, o trabalho [Das and Bhattacharyya 2015] apresenta um esquema geração de rotas que minimiza a distância, considerando veículos de tração humana e automotores. Os trabalhos [Vu et al. 2018, Das and Bhattacharyya 2015] não avaliaram o tempo de percurso e esforço físico dos carrinheiros de acordo com as rotas geradas.

Dentre os trabalhos que abordam a definição de rotas para minimizar distâncias na coleta seletiva, destaca-se [Ahmad et al. 2020], que propõe um sistema ideal de recomendação de roteiros para diminuir a distância percorrida, o consumo de combustível do caminhão e maximizar a quantidade de materiais recolhidos. É possível citar também o trabalho [Benjamin and Beasley 2010], o qual propõe uma solução considerando janelas de tempo, período de descanso dos condutores e múltiplas instalações de disposição de resíduos. Por fim, o trabalho [Tavares et al. 2009] propõe um modelo para otimização de rotas, minimizando o consumo de combustível dos veículos que coletam e transportam materiais recicláveis.

Outros trabalhos sugerem algoritmos heurísticos para gerar rotas otimizadas considerando veículos automotores, a fim de diminuir a distância percorrida, minimizar os custos financeiros e o consumo de combustível. Sendo assim, o trabalho [Liao and Ting 2010] apresenta uma solução logística adaptada à restrição de carga, na qual se deve coletar mercadorias de alguns vértices e cumprir todas as respectivas entregas. Ademais, o trabalho [Huang and Ting 2011] propõe um algoritmo com restrições de carga e distância máxima a ser percorrida por cada veículo de uma frota, minimizando a distância total de todos os veículos. Nesse sentido, os trabalhos relacionados estão, em sua maioria, focados no roteamento para veículos automotores. É necessário considerar a utilização de veículos de tração humana no roteamento para coleta seletiva, uma vez que os carrinheiros são fundamentais neste processo, especialmente em países emergentes.

\section{Tecnologias na coleta de resíduos}

Os carrinheiros geralmente trabalham vinculados a cooperativas ou de forma independente. Nas cooperativas, estão inseridos na metodologia de coleta de três estágios. Esta metodologia pode ser descrita a partir dos processos empregados por uma cooperativa na cidade de Belém, no estado do Pará, Brasil: o caminhão leva os carrinheiros com os veículos de tração humana até um local pré-determinado da cidade. A partir deste local, cada carrinheiro segue uma rota a pé, etapa que também é chamada de coleta primária. A Figura 1 apresenta uma carrinheira durante esta fase. Em seguida, o caminhão transporta os carrinheiros e os resíduos coletados até o galpão de triagem. Após a classificação dos materiais, um veículo de grande porte leva os resíduos até as indústrias de reciclagem.

Na coleta primária, os carrinheiros recolhem os materiais recicláveis na casa das pessoas e estabelecimentos comerciais. Estes locais de parada do roteiro são chamados 


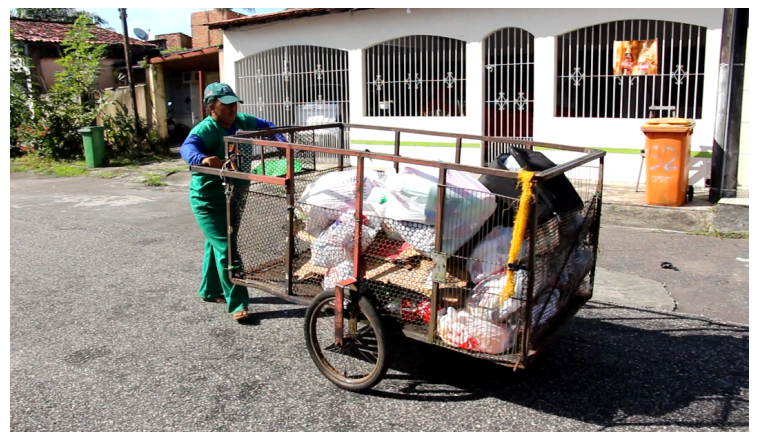

Figura 1. Carrinheira utilizando veículo de tração humana na coleta primária

de pontos de coleta. Sendo assim, para que o recolhimento dos materiais aconteça de forma eficiente, o catador precisa saber a localização destes locais previamente. Existem tecnologias que possibilitam identificar os pontos de coleta, como por exemplo o Cataki [CATAKI 2021] e o Destino Sustentável [Oliveira et al. 2019].

Os aplicativos Cataki e Destino Sustentável possibilitam solicitar e agendar coleta de materiais recicláveis com catadores (incluindo carrinheiros). Para isso, o solicitante precisa informar o tipo de resíduo, a quantidade/peso dos materiais, os dias e horários disponíveis para entregar os materiais ao catador, assim como o local de entrega. No Cataki, ao solicitar a coleta, o aplicativo indica um catador geograficamente próximo para recolher os materiais no local cadastrado. Após selecionar o(a) catador(a), a coleta fica registrada na agenda de quem vai coletar e do solicitante. No Destino Sustentável, este processo ocorre de maneira parecida. Porém, após solicitar a coleta, um catador precisa atender o anúncio para que o recolhimento do resíduo seja agendado [Oliveira et al. 2019].

Com a agenda definida a partir do aplicativo Cataki ou Destino Sustentável, os catadores podem decidir o roteiro de coleta, visto que é possível identificar previamente os materiais que serão recolhidos, a quantidade e a localização dos pontos de parada. Nesse sentido, é preciso definir a ordem de visitação desses pontos considerando o acréscimo de peso ao veículo em cada ponto de coleta. Um carrinheiro vinculado a uma cooperativa considerada de alta eficiência recolhe, em média, 85,7 quilos de resíduos por dia [Silva 2017]. Nessa perspectiva, muitas vezes os carrinheiros criam roteiros baseados no conhecimento empírico do local para minimizar o esforço físico durante o trajeto.

\section{Algoritmo e políticas implementadas}

Esta seção descreve o algoritmo proposto e as políticas implementadas. O algoritmo proposto gera rotas otimizadas utilizando uma das políticas (PMT, PMI e PMD). Estas políticas descrevem o mecanismo de atualização de peso das arestas dos grafos. Sendo assim, é possível dividir o algoritmo proposto nas seguintes etapas: cálculo da área que compreende o trajeto; obtenção dos dados geográficos; construção dos grafos; ordenação das visitas aos pontos de coleta e criação da rota.

Dado um conjunto de coordenadas geográficas, a primeira representa o ponto de início e a última, o destino final. As outras coordenadas representam os pontos de coleta. Com base nisso, o algoritmo delimita uma área retangular com os valores mínimos e máximos de latitude e longitude do conjunto de coordenadas. Esta área é utilizada para obtenção de dados geográficos do Open Street Map e de altitude, em formato GeoTiff, no 
Banco de Dados Geomorfométricos, do projeto Topodata [Valeriano and Rossetti 2012]. Em seguida, emprega-se a ferramenta osmnx para gerar o grafo da região delimitada, chamado grafo geográfico. Nesse caso, os vértices representam as esquinas e as arestas, as ruas [Boeing 2017]. Depois, identifica-se os valores de altitude de cada vértice do grafo geográfico para configurar os ângulos das arestas.

Os dados geográficos do Open Street Map adicionados no grafo são as coordenadas dos vértices, a velocidade máxima das vias, o tipo de pavimentação e o tamanho das arestas. Vias com velocidades máximas de $40 \mathrm{Km} / \mathrm{h}$ ou menos são configuradas como bidirecionais, possibilitando ao carrinheiro andar nos dois sentidos da rua. Além disso, os pontos de parada são adicionados no grafo, sendo que o primeiro ponto é chamado de vértice de início e o último, de vértice depósito (local de disposição dos materiais).

Para definir a ordem de visitação dos pontos de coleta, cria-se um grafo completo utilizando o networkx, nomeado de grafo para ordenação, com o número de vértices igual ao número de pontos de parada mais os pontos inicial e final. Por ser completo, cada vértice é adjacente a todos os outros. Nesse sentido, o custo das arestas do grafo para ordenação é calculado a partir do trajeto entre os pontos de coleta, no grafo geográfico. A Figura 2(a) ilustra um exemplo de grafo para ordenação, com dois pontos de coleta destacados. O custo da aresta entre os vértices 8 e 9 é calculado de acordo com o custo total do caminho entre os dois pontos de coleta no grafo geográfico. A Figura 2(b) apresenta a rota que conecta os vértices 8 e 9 no grafo geográfico destacada em vermelho.

Os caminhos no grafo geográfico são definidos a partir do algoritmo SPFA, baseado no algoritmo de Bellman-Ford. Este algoritmo foi escolhido porque aceita pesos negativos de arestas [Moore 1959, Madkour et al. 2017]. Além disso, a ordenação dos pontos de coleta é realizada utilizando a heurística do Vizinho Mais Próximo, que é amplamente utilizada em problemas de roteamento de veículos, possui baixo tempo de processamento e bom desempenho [Daanish and Naick 2017].

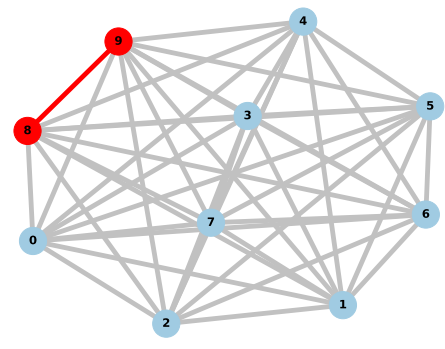

(a) Grafo para ordenação

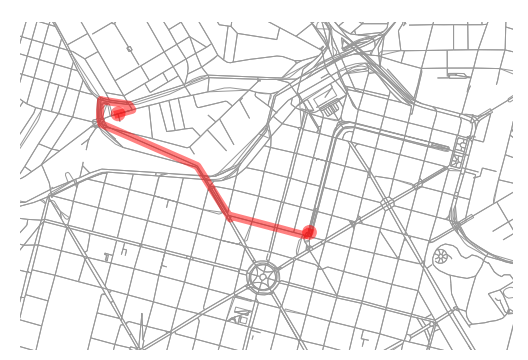

(b) Grafo geográfico

Figura 2. llustração dos grafos utilizados para determinação da rota

Descreve-se a definição das rotas nas seguintes etapas: partindo-se do nó inicial, verifica-se o custo desse nó até cada um dos vértices adjacentes que não foram visitados ainda. Esse custo é calculado de acordo com algoritmo SPFA, que escolhe o melhor caminho entre o par de vértices, no grafo geográfico. Em seguida, encontra-se o vértice mais próximo do nó atual, ou seja, que possui o menor custo. O nó mais próximo passa a ser o vértice atual e, novamente, calcula-se os custos até os vértices que não foram visitados. Esse processo é realizado até todos que os pontos de coleta sejam inseridos no caminho. Visto que o nó depósito sempre será o último vértice, ele é inserido no final. 
O mecanismo de geração de rotas está descrito mais detalhadamente no Algoritmo 1. Além disso, a Tabela 1 apresenta um sumário dos símbolos utilizados e seus respectivos significados.

Tabela 1. Sumário dos símbolos e significados utilizados nesta seção

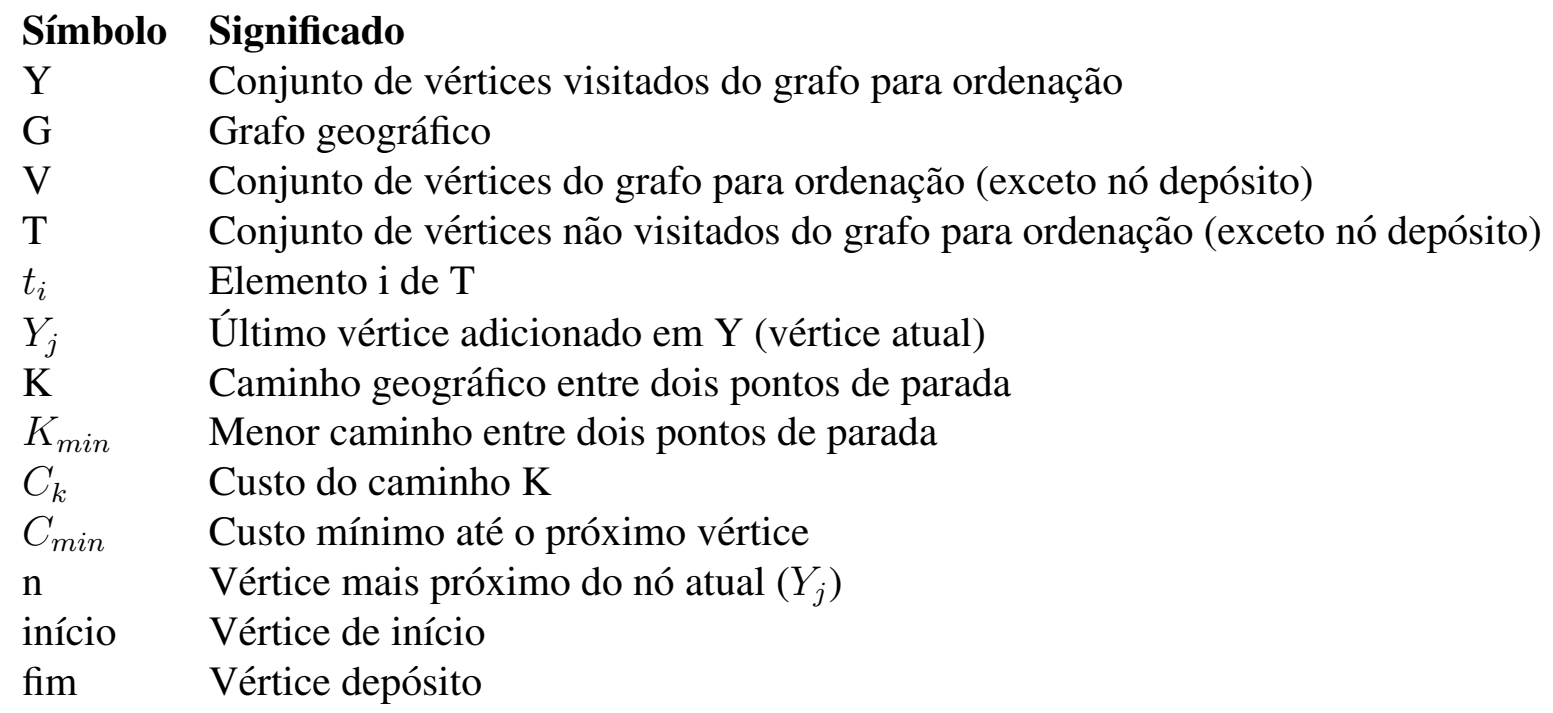

O Algoritmo 1 descreve a combinação da heurística Vizinho Mais Próximo com o algoritmo SPFA. Os parâmetros de entrada do Algoritmo 11 são: vértice inicial (início), vértice final (vértice depósito), o grafo geográfico $(G)$; conjunto de vértices do grafo para ordenação $(V)$; conjunto de vértices que ainda não foram visitados do grafo para ordenação (T); a política (PMT, PMI ou PMD) e a massa inicial do veículo.

De acordo com o Algoritmo 1, enquanto o conjunto de vértices visitados $(Y)$ for diferente do conjunto de vértices do grafo para ordenação $(V)$ (linha 4), configura-se o custo mínimo até o nó mais próximo $\left(C_{\min }\right)$ igual a infinito (linha 5). Para cada vértice não visitado $t_{i}$ (linha 6), identifica-se o caminho $(K)$ entre o nó atual $\left(Y_{j}\right)$ e $t_{i}$. Este caminho é calculado por meio do algoritmo SPFA (linha 7). Se o custo do caminho $(C)$ for menor que o custo mínimo $\left(C_{\min }\right)$, atualiza-se o custo mínimo $\left(C_{\min }\right)$ (linha 8), o menor caminho $\left(K_{\min }\right)$ e o nó mais próximo $(n)$ (linhas 10 e 11$)$.

Depois de identificar o vértice mais próximo $(n)$ do nó atual $\left(Y_{j}\right)$, adiciona-se o menor caminho $\left(K_{\min }\right)$ na rota (linha 14$)$. Em seguida, o nó mais próximo $(n)$ é acrescentado ao conjunto de vértices visitados $(Y)$ (linha 15), tornando-se o vértice atual $\left(Y_{j}\right)$. Além disso, se a política utilizada for a PMT, a massa do veículo é atualizada de acordo com a função atualizaMassa. Esta função verifica qual o valor de massa dos materiais que serão coletados no ponto de coleta $n$ e acrescenta este valor à massa do veículo atual (linha 17). Ademais, atualiza-se os pesos das arestas do grafo geográfico a partir do novo valor de massa do veículo (linha 18). Quando todos os vértices contidos em $V$ forem visitados, cria-se o caminho do último nó adicionado $\left(Y_{j}\right)$ até o nó depósito (linha 21). Acrescenta-se este caminho na rota (linha 22) e, por fim, o algoritmo retorna a rota. O código completo do algoritmo está disponível no GitHub 1 .

O Algoritmo 1 apresentou o processo de geração de rotas utilizando a heurística

\footnotetext{
${ }^{1}$ https://github.com/vivirodrigues/Carrinheiros
} 


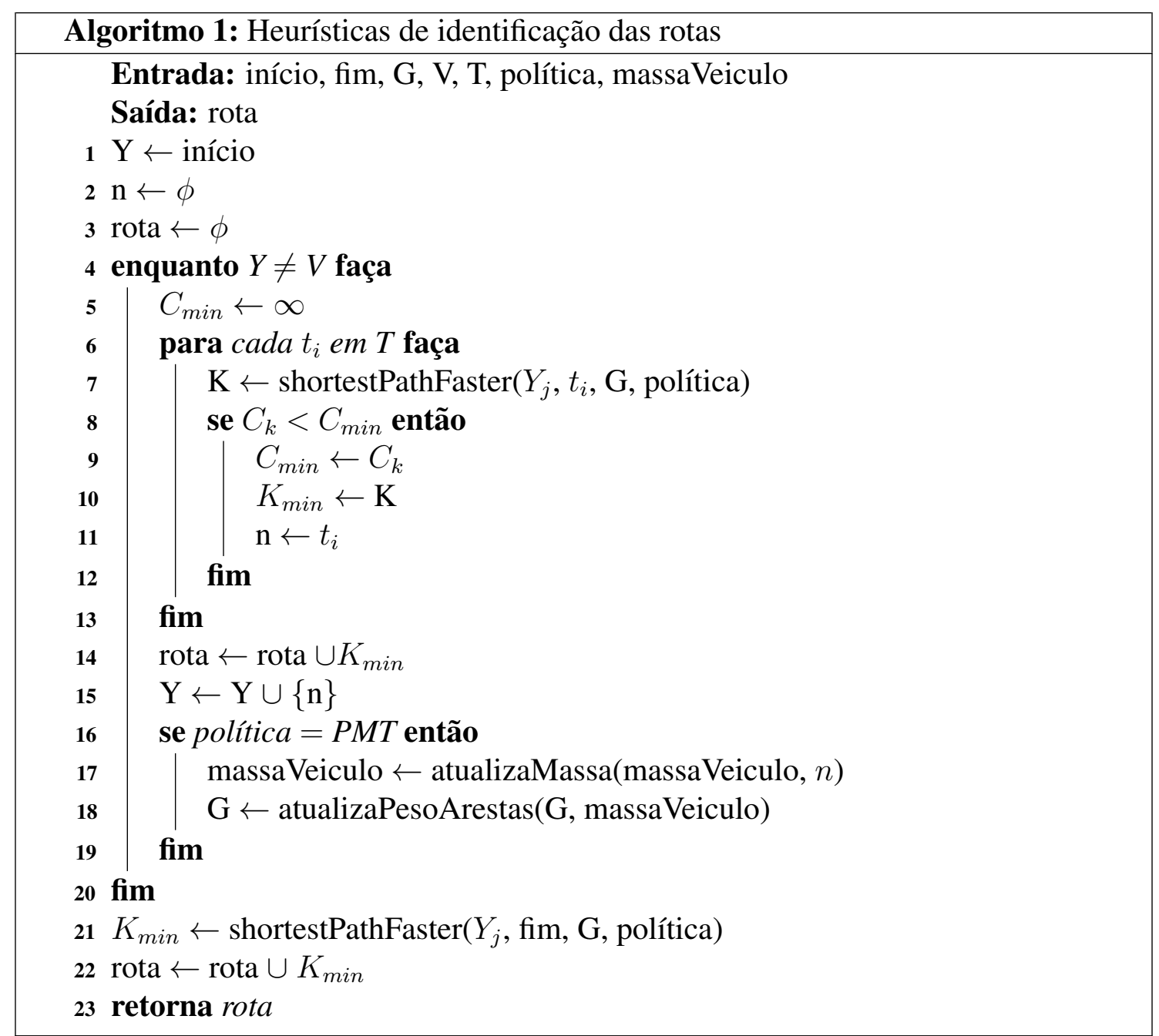

do Vizinho Mais Próximo e o algoritmo SPFA. Além disso, foi possível identificar que a política PMT atualiza o peso do veículo e das arestas cada vez que um nó é visitado no grafo para ordenação.

\subsection{Política de Menor Trabalho}

A política PMT calcula o peso das arestas do grafo geográfico a partir do trabalho necessário para empurrar o veículo em determinada aresta. De acordo com a mecânica newtoniana, este cálculo pode ser realizado por meio da equação 1 .

$$
W=\left[\left(m * g * f_{r} \cos \theta\right)+(m * g * \sin \theta)+\left(\frac{1}{2} * \rho * C * S * v^{2}\right)\right] * d
$$

Na qual $W$ representa o valor do Trabalho, $m$ o valor da massa do veículo, $g$ a constante de aceleração da gravidade, $f_{r}$ o coeficiente de resistência ao rolamento, $\theta$ o ângulo de inclinação da via, $\rho$ a densidade do ar, $C$ corresponde ao coeficiente de arrasto, $S$ a área frontal do veículo, $v$ a velocidade do ar em relação ao corpo e $d$ representa a distância total da aresta [Silveira 2011] [Halliday et al. 2016]. 
A primeira componente da equação corresponde à força de resistência ao rolamento, associada à perda de energia mecânica na roda, que ocorre em razão da deformação do pneu em contato com o solo. Nesse caso, o valor de $f_{r}$ depende do tipo de roda do veículo e da pavimentação. Além disso, a segunda componente representa a força de ação da gravidade, a qual tem influência resistente em aclives e adjuvante em declives. Na terceira componente, tem-se a força de resistência do ar em relação ao veículo em movimento, chamada de força de arrasto. Considera-se que a velocidade do ar em relação ao corpo $(v)$ é igual a velocidade do carrinho, consequentemente, o ar está parado em relação à via [Silveira 2011].

\subsection{Política de Menor Impedância}

A política PMI evita aclives acentudados e foi idealizada para ser utilizada quando os carrinheiros possuem limitações físicas ou preferem caminhos com poucas subidas. Nesse sentido, propõe-se calcular o valor da Impedância $(I)$ de acordo com a equação 2 .

$$
I= \begin{cases}\theta^{2} * d, & \text { se } \theta>0 \\ \theta *(-1) * d, & \text { caso contrário }\end{cases}
$$

Na qual $\theta$ representa o ângulo da via e $d$, o tamanho da aresta (distância). A partir da equação 2, tem-se um valor constante de peso das arestas, pois não precisa ser atualizado durante o percurso, diferentemente do que ocorre com a política PMT.

\subsection{Política de Menor Distância}

A política PMD minimiza a distância percorrida. Neste caso, o peso das arestas é igual ao valor da distância/tamanho da aresta, obtida por meio do Open Street Map.

\section{Resultados}

Esta Seção apresenta os parâmetros utilizados nas simulações e os resultados obtidos.

\subsection{Parâmetros das simulações}

Dois cenários foram utilizados para validar a proposta, com diferentes variações de altitude, ambos no Brasil. O primeiro é na cidade de Belo Horizonte, no estado de Minas Gerais, e o segundo é na cidade de Belém, no estado do Pará. Ilustra-se os grafos dos cenários de Belo Horizonte na Figura 3(a) e Belém na Figura 3(b), a partir dos dados de altitude normalizada. De acordo com a Figura 3(a), é possível perceber que a elevação varia de 0 a 150 metros no cenário de Belo Horizonte. Na Figura 3(b), que representa o cenário de Belém, a elevação varia de 0 a 30 metros. Nesse sentido, o cenário de Belo Horizonte possui maior variação na altitude dos nós.

As rotas foram geradas pelo algoritmo proposto e validadas no simulador Simulation of Urban MObility (SUMO). De acordo com a Tabela 2, que demonstra os parâmetros das simulações, utilizou-se 8 pontos de coleta em cada simulação. Nesses pontos, a massa do veículo é incrementada de forma pseudo-aleatória, com valor máximo de $50 \mathrm{Kg}$. Essa atualização da massa do veículo representa os materiais recicláveis recolhidos. Além dos pontos de coleta, define-se os pontos inicial e final da rota. As coordenadas $x$ e $y$ dos pontos seguem uma distribuição gaussiana com desvio padrão 0,005 , média 


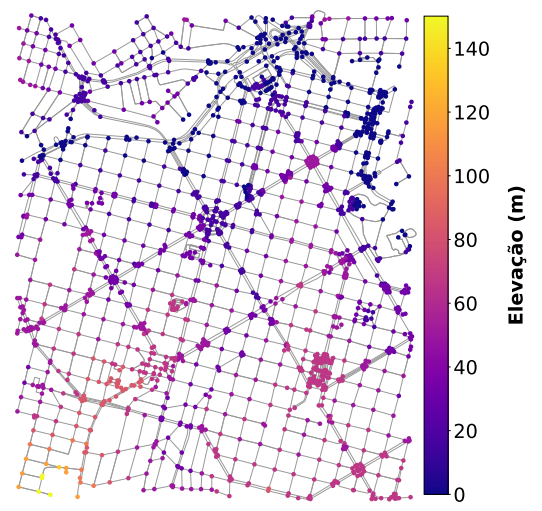

(a) Cenário Belo Horizonte

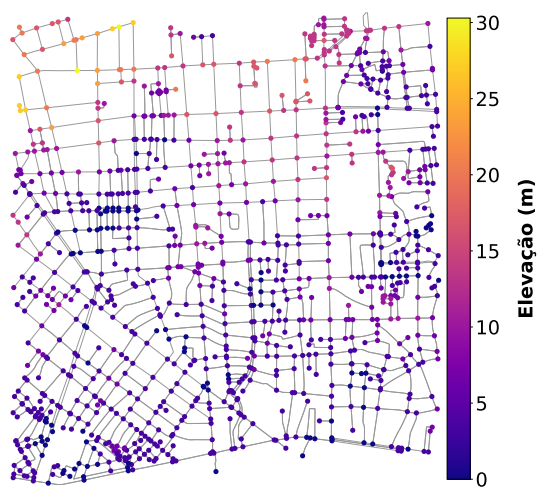

(b) Cenário Belém

Figura 3. Cenários de simulação

(-43,9438, -19,9202) no cenário de Belo Horizonte e média $(-48,47000,-1,46000)$, no cenário de Belém. A massa inicial do veículo foi configurada como $110 \mathrm{Kg}$.

Para avaliar os resultados, escolheu-se as métricas: potência aplicada para empurrar o veículo, distância total do trajeto e tempo de percurso. O cálculo da potência instantânea foi realizado a partir da equação 3 [Silveira 2011] e implementado na simulação por meio da ferramenta Traci do simulador SUMO. As simulações foram executadas 30 vezes utilizando diferentes sementes de aleatoriedade e intervalo de confiança de $95 \%$.

$$
P=\left[\left(m * g * f_{r} \cos \theta\right)+(m * g * \sin \theta)+\left(\frac{1}{2} * \rho * C * S * v^{2}\right)\right] * v
$$

Na qual $P$ representa a potência instantânea, $m$ o valor da massa do veículo, $g$ a constante de aceleração da gravidade, $f_{r}$ o coeficiente de resistência ao rolamento, $\theta$ o ângulo de inclinação da via, $\rho$ a densidade do ar, $C$ corresponde ao coeficiente de arrasto, $S$ a área frontal do veículo e $v$ a velocidade instantânea do veículo, que é definida automaticamente pelo algoritmo do simulador SUMO. Como mostra a Tabela 2, configurou-se a velocidade máxima do veículo como $3,6 \mathrm{Km} / \mathrm{h}$, a área frontal do veículo $1 \mathrm{~m}^{2}$, a densidade do ar $1,2 \mathrm{Km} / \mathrm{m}^{3}$ e o coeficiente aerodinâmico 1 . Por fim, os coeficientes de resistência ao rolamento foram definidos de acordo com o tipo de pavimentação das vias [Costa 2016].

\subsection{Potência aplicada no veículo}

A potência aplicada no veículo depende da força de tração e da velocidade empregada pelo carrinheiro. Visto que é necessário aplicar esforço físico para locomover os veículos, os trajetos de coleta são mais cansativos quando se emprega altas potências. Sendo assim, a Figura 4 apresenta os resultados da potência média empregada no veículo conforme as simulações das rotas geradas pelo algoritmo proposto, utilizando as políticas PMT, PMI e PMD.

De acordo com a Figura 4, os valores de potência média aplicada no veículo das rotas geradas considerando o cenário de Belo Horizonte foram 46,7 W, 45,5 e 82,7 W, utilizando as políticas PMT, PMI e PMD, respectivamente. As rotas geradas pelo algoritmo 
Tabela 2. Parâmetros utilizados nas simulações

\section{Parâmetro}

Número de pontos de coleta por simulação

Incremento máximo de massa do veículo

Média das coordenadas x e y Belo Horizonte

Média das coordenadas x e y Belém

Desvio padrão das coordenadas

Velocidade máxima do carrinheiro

Peso inicial do veículo

Área frontal do veículo

Densidade do ar

Coeficiente aerodinâmico

Aceleração da gravidade
Valor

8

$50 \mathrm{Kg}$

$(-43,9438,-19,9202)$

$(-48,47000,-1,46000)$

0,005

$3,6 \mathrm{Km} / \mathrm{h}$

$110 \mathrm{Kg}$

$1 \mathrm{~m}^{2}$

$1,2 \mathrm{Km} / \mathrm{m}^{3}$

1

9,80665

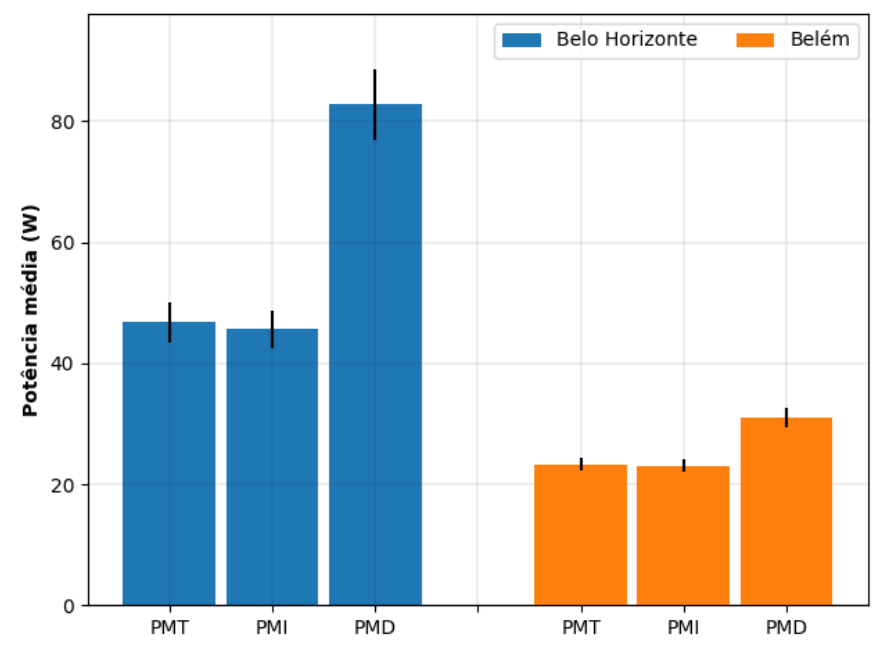

Figura 4. Potência média empregada para empurrar o veículo

no cenário de Belém empregando as políticas PMT, PMI e PMD alcançaram valores de potência média de $23,3 \mathrm{~W}, 23,06 \mathrm{~W}$ e $31,08 \mathrm{~W}$, respectivamente.

Os resultados da Figura 4 demonstram que os valores de potência média aplicada no veículo no cenário de Belém foram menores do que no cenário de Belo Horizonte, que possui maior variação de altitude. Verifica-se, então, que os aclives e declives influenciam na potência média aplicada no veículo. O algoritmo gerou rotas com valores semelhantes de potência média aplicada no veículo utilizando as políticas PMT e PMI, tanto no cenário de Belo Horizonte, quanto Belém. Sendo assim, considerar a declividade do local na escolha do trajeto diminuiu a potência média empregada para empurrar o veículo. As rotas geradas pelo algoritmo utilizando a política PMI obtiveram os maiores valores de potência média nos dois cenários. Portanto, as rotas que priorizaram a menor distância de trajeto exigiram maior esforço físico nos cenários implementados.

Além da potência média, é preciso analisar os valores de potência instantânea aplicada no veículo durante as simulações. Para isso, a Figura 5(a) e a Figura 5(b) apresentam os resultados da Função de Distribuição Acumulada (FDA) Empírica da potência 
instantânea aplicada no veículo nos cenários de Belo Horizonte e Belém.

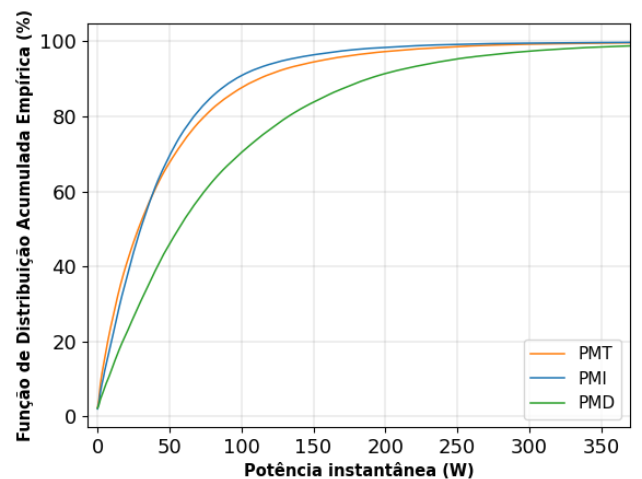

(a) Belo Horizonte

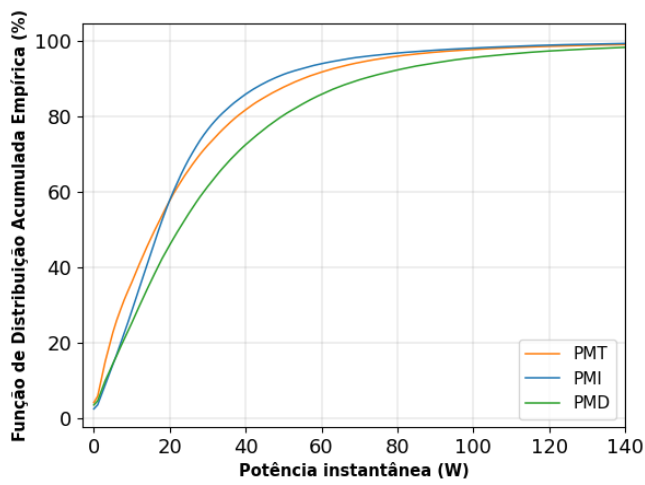

(b) Belém

Figura 5. Função de Distribuição Acumulada Empírica da potência instantânea aplicada

De acordo com os resultados da Figura 5(a), as rotas geradas pelo algoritmo utilizando a política PMI proporcionaram 69\% de probabilidade de aplicar valores menores ou iguais a $50 \mathrm{~W}$ de potência instantânea no veículo e $90 \%$ de probabilidade de aplicar potências instantâneas menores ou iguais a $100 \mathrm{~W}$. As rotas geradas pelo algoritmo utilizando a política PMT apresentaram resultados parecidos com as rotas da política PMI: $67 \%$ de probabilidade do valor de potência aplicada no veículo ser menor ou igual a $50 \mathrm{~W}$ e $87 \%$ de probabilidade de ser menor ou igual a $100 \mathrm{~W}$. Além disso, as rotas geradas pela política PMD obtiveram $45 \%$ e $70 \%$ de probabilidade de aplicarem potências instantâneas menores ou iguais a $50 \mathrm{~W}$ e $100 \mathrm{~W}$, respectivamente.

Observa-se na Figura 5(a) que a FDA empírica se aproxima de 100\% a partir do valor $250 \mathrm{~W}$ nas políticas PMT e PMI, enquanto que na política PMD, a FPA empírica se aproxima de $100 \%$ após $350 \mathrm{~W}$. Além disso, os resultados das simulações utilizando a política PMI demonstraram menor probabilidade do veículo apresentar potências instantâneas mais baixas. Considera-se, então, que as rotas dessa configuração exigem maior esforço físico do carrinheiro para empurrar o veículo.

Os resultados da Figura 5(b) demonstram que a política PMT proporcionou a obtenção de rotas com $87 \%$ de probabilidade de empregar $50 \mathrm{~W}$ ou menos de potência instantânea no veículo e $97 \%$ de probabilidade de aplicar potências menores ou iguais a 100 W. Além disso, as rotas geradas com a política PMI apresentaram $91 \%$ de probabilidade de aplicar $50 \mathrm{~W}$ ou menos de potência instantânea no veículo e $98 \%$ de probabilidade de aplicar valores menores ou iguais a $100 \mathrm{~W}$. Por fim, as rotas geradas pelo algoritmo adotando a política PMD apresentaram $80 \%$ de probabilidade de empregar valores menores ou iguais a $50 \mathrm{~W}$ de potência instantânea no veículo e $95 \%$ de probabilidade de aplicar potências instantâneas menores ou iguais a $100 \mathrm{~W}$.

Em conformidade com os resultados de potência média apresentados na Figura 4 , as rotas geradas utilizando as políticas PMT e PMI no cenário com menor variação de altitude alcançaram resultados aproximados. Além disso, a variação de potência instantânea aplicada no cenário Belém é de 0 a aproximadamente $140 \mathrm{~W}$. Portanto, há menor variação 
de potência aplicada neste cenário, em comparação com o cenário de Belo Horizonte, que é aproximadamente $350 \mathrm{~W}$.

\subsection{Tempo de trajeto e distância total do roteiro}

A avaliação da distância e tempo total do trajeto permitem identificar a política mais adequada para os carrinheiros que preferem rotas mais curtas e rápidas, garantindo a personalização das rotas. Com base nos resultados das simulações, a Figura 6(a) apresenta a distância total dos roteiros e a Figura 6(b) demonstra os resultados do tempo de trajeto.

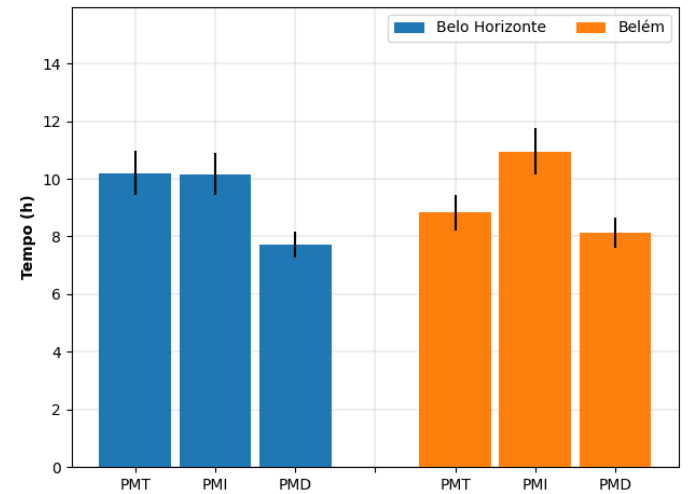

(a) Distância

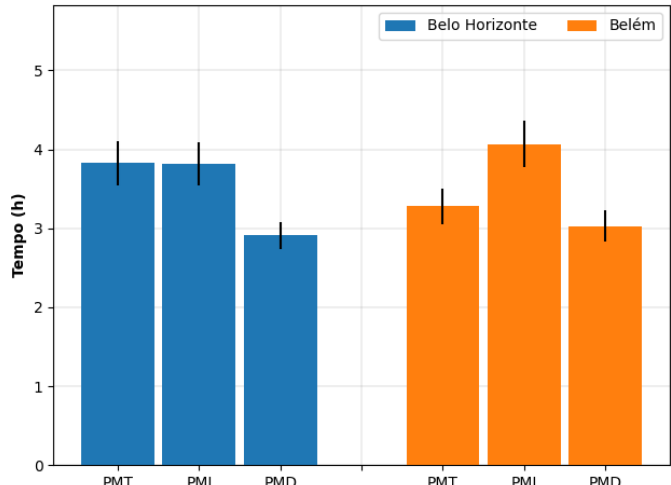

(b) Tempo

Figura 6. Distância e Tempo de trajeto

De acordo com a Figura 6(a), as médias de distância percorrida na simulação das rotas geradas no cenário de Belo Horizonte foram 10,2 Km, 10,16 Km e 7,7 Km, empregando as políticas PMT, PMI e PMD, respectivamente. Enquanto que no cenário de Belém, o algoritmo produziu rotas com distância de $8,8 \mathrm{Km}, 10,9 \mathrm{Km}$ e $8,1 \mathrm{Km}$, utilizando as políticas PMT, PMI e PMD, respectivamente. Em seguida, a Figura 6(b) apresenta a comparação entre as médias de tempo de trajeto das rotas geradas pelo algoritmo nos dois cenários. As médias do tempo de percurso das rotas geradas no cenário de Belo Horizonte foram 3,8, 3,8 e 2,9 horas utilizando as políticas PMT, PMI e PMD, respectivamente. Além disso, no cenário de Belém, as rotas geradas alcançaram 3,2, 4,06 e 3,02 horas com as políticas PMT, PMI e PMD, respectivamente.

As rotas geradas utilizando a política PMD obtiveram a menor média de tempo e distância nos dois cenários. Sendo assim, os carrinheiros que desejam realizar trajetos rápidos podem empregar a política PMD. Para evitar vias que demandam maior esforço físico, como aclives acentuados, as políticas PMT e PMI proporcionaram rotas um pouco mais longas no cenário de Belo Horizonte. Porém, o tempo de trajeto e distância percorrida das rotas geradas com a política PMT no cenário de Belém foram menores em comparação com a política PMI. Portanto, no cenário de menor variação de altitude (Belém), a política PMT proporciona rotas que minimizam eficientemente a potência média, além de alcançar rotas mais rápidas e menores do que a política PMI. Então, a política PMT minimiza eficientemente o esforço físico dos carrinheiros. 


\section{Conclusão}

Este trabalho apresentou uma abordagem original de sugestão de rotas para catadores que utilizam veículos de propulsão humana na coleta seletiva, desenvolvida a partir das tecnologias livres networkx, osmnx e Open Street Map. O algoritmo proposto gera rotas de coleta utilizando a heurística do Vizinho mais Próximo para ordenar os pontos de coleta e o algoritmo SPFA, para determinar o melhor caminho entre pontos de coleta. Além disso, três políticas de personalização de rotas foram propostas: Política de Minimização do Trabalho, Política de Minimização da Impedância e Política de Minimização da Distância.

A política PMT obteve o melhor desempenho quanto à minimização do esforço físico porque, além de apresentar bom desempenho na diminuição da potência aplicada no veículo, proporcionou rotas com menor distância e tempo, em comparação com a política PMI, no cenário de Belém. Além disso, as rotas mais curtas e rápidas foram obtidas a partir da política PMD. No entanto, estas rotas apresentaram os maiores valores de potência aplicada. Demonstrando, assim, que os caminhos de menor distância não foram os que diminuíram o esforço físico do carrinheiro nos cenários considerados.

Este trabalho apresenta relevância social, pois possibilita melhorar a qualidade de vida dos carrinheiros por meio de sugestões de rotas que minimizam o esforço físico. Como trabalhos futuros, propõe-se sugestões de rotas que evitam vias de tráfego em alta velocidade, para minimizar os riscos de acidentes. Além disso, propõe-se o desenvolvimento de um algoritmo de geração de rotas para múltiplos veículos com restrições de tempo e capacidade, de modo que o tempo de percurso dos trajetos seja parecido e os carrinheiros possam retornar ao ponto de chegada ao mesmo tempo.

\section{Referências}

Ahmad, S., Imran, Jamil, F., Iqbal, N., and Kim, D. (2020). Optimal route recommendation for waste carrier vehicles for efficient waste collection: A step forward towards sustainable cities. IEEE Access, 8:77875-77887.

Benjamin, A. M. and Beasley, J. E. (2010). Metaheuristics for the waste collection vehicle routing problem with time windows, driver rest period and multiple disposal facilities. Computers and Operations Research, 37(12):2270-2280.

Boeing, G. (2017). Osmnx: New methods for acquiring, constructing, analyzing, and visualizing complex street networks. Computers Environment and Urban Systems, 65:126-139.

Boskovic, G. and Jovicic, N. (2015). Fast methodology to design the optimal collection point locations and number of waste bins: A case study. Waste Management \& Research, 33(12):1094-1102.

BRASIL (2010). Lei ${ }^{\circ}$ 12.305, de 2 de agosto de 2010. Institui a Política Nacional de Resíduos Sólidos e outras providências.

CATAKI (2021). https://www.cataki.org/pt/. Acessado em: 2021-04-04.

Costa, F. A. M. (2016). Proposta de mecanismo: motor pneumático para veículo de coleta de recicláveis. In Monografia do Bacharelado em Engenharia Mecânica, Universidade de Brasília, pages 1-105. 
Daanish, A. R. and Naick, B. K. (2017). Implementation of charging station based electric vehicle routing problem using nearest neighbour search algorithm. In 2017 2nd IEEE International Conference on Intelligent Transportation Engineering (ICITE), pages 52-56. IEEE.

Das, S. and Bhattacharyya, B. K. (2015). Optimization of municipal solid waste collection and transportation routes. Waste Management, 43:9-18.

Gallardo, A., Carlos, M., Peris, M., and Colomer, F. (2015). Methodology to design a municipal solid waste pre-collection system. a case study. Waste management, 36:111.

Halliday, D., Resnick, R., and Walker, J. (2016). Fundamentos de Física, volume 1: Mecânica. Ed. LTC, Rio de Janeiro.

Huang, Y. H. and Ting, C. K. (2011). Genetic algorithm with path relinking for the multivehicle selective pickup and delivery problem. 2011 IEEE Congress of Evolutionary Computation, CEC 2011, pages 1818-1825.

Liao, X. L. and Ting, C. K. (2010). An evolutionary approach for the selective pickup and delivery problem. IEEE Congress on Evolutionary Computation.

Madkour, A., Aref, W. G., Rehman, F. U., Rahman, M. A., and Basalamah, S. (2017). A survey of shortest-path algorithms. arXiv preprint arXiv:1705.02044.

Moore, E. (1959). The Shortest Path Through a Maze. Bell Telephone System. Technical publications. monograph. Bell Telephone System.

Oliveira, M., Silva, J., Silva, R., and Teran, L. (2019). Aplicação web para gerenciamento de residuos sólidos recicláveis. In Anais do X Workshop de Computação Aplicada a Gestão do Meio Ambiente e Recursos Naturais, pages 145-153, Porto Alegre, RS, Brasil. SBC.

Rathore, P., Sarmah, S. P., and Singh, A. (2020). Location-allocation of bins in urban solid waste management: a case study of bilaspur city, india. Environment, Development and Sustainability, 22(4):3309-3331.

Silva, S. P. (2017). A organização coletiva de catadores de material reciclável no brasil: dilemas e potencialidades sob a ótica da economia solidária. In Instituto de Pesquisa Econômica Aplicada (Ipea), pages 1-56.

Silveira, F. L. d. (2011). Potência de tração de um veículo automotor que se movimenta com velocidade constante. Revista Brasileira de Ensino de Física, 33(1):01-07.

Tavares, G., Zsigraiova, Z., Semiao, V., and Carvalho, M. (2009). Optimisation of msw collection routes for minimum fuel consumption using $3 \mathrm{~d}$ gis modelling. Waste Management, 29(3):1176-1185.

Valeriano, M. d. M. and Rossetti, D. d. F. (2012). Topodata: Brazilian full coverage refinement of srtm data. Applied Geography, 32(2):300-309.

Vu, H. L., Ng, K. T. W., and Bolingbroke, D. (2018). Parameter interrelationships in a dual phase gis-based municipal solid waste collection model. Waste Management, 78:258-270. 